Copyright (C 2014 IEEE. Personal use of this material is permitted. Permission from IEEE must be obtained for all other uses, in any current or future media, including reprinting/republishing this material for advertising or promotional purposes, creating new collective works, for resale or redistribution to servers or lists, or reuse of any copyrighted component of this work in other works. 


\title{
Developing the Guidelines for Fabrication of Laboratory Prototype Voltage Source Converters
}

\author{
Ruwan P.S. Chandrasena, Florian Barbieri, Farhad Shahnia, Arindam Ghosh and Sumedha Rajakaruna \\ Electrical and Computer Engineering Department \\ Curtin University \\ Perth, Australia \\ ruwan.chandrasena@curtin.edu.au
}

\begin{abstract}
This paper presents the general guidelines on developing a laboratory prototype Voltage Source Converter (VSC). One of the main possible reasons behind the limited hardware verification by postgraduate students, carrying out research in the area of power electronics and especially the VSCs, is the lack of knowledge and unavailability of proper tutorials and guidelines for developing hardware prototypes. In this paper, majority of the auxiliary circuits and modules which are required for building up a prototype VSC are introduced. Proper examples are provided at each stage to improve the effectiveness of the developed guideline. The main difficulties of the hardware experiments are mentioned and possible solutions and recommendations are presented throughout the paper. It is believed that this paper will highly benefit the postgraduate students at the early stages of their hardware experiments.
\end{abstract}

Index Terms-Voltage Source Converter (VSC), Laboratory prototype, Printed Circuit Board (PCB), Printed Circuit Board Assembly (PCBA).

\section{INTRODUCTION}

Voltage Source Converters (VSC) are used widely in power systems nowadays. They may range from simple regular single-phase and three-phase converters to more complex topologies such as multi-level converters. VSCs can be used for power conditioning as well as power transfer control in transmission and distribution systems, integration of distributed generation units with power systems, High Voltage Direct Current (HVDC) transmission systems and electric motor drives [1-4]. One of the major research topics in recent years is utilizing VSCs for newer applications and developing modified topologies and improved control systems to increase the VSC efficiency, design and dynamic performance. As an example, by a key word search on "voltage source converters" in IEEExplore, it can be seen that 203 research papers have been published in different IEEE transactions in the last 10 years. However, only about $20 \%$ of them have been experimentally verified.

Limited number of publication with experimental verification is linked to several reasons. Building a hardware setup costs money. In addition, it needs some hardware building skills and technical knowhow about handling the electrical and electronic devices and components. Furthermore, it takes time to build a hardware test rig since it has to go through various stages such as ordering components, building PCBs,

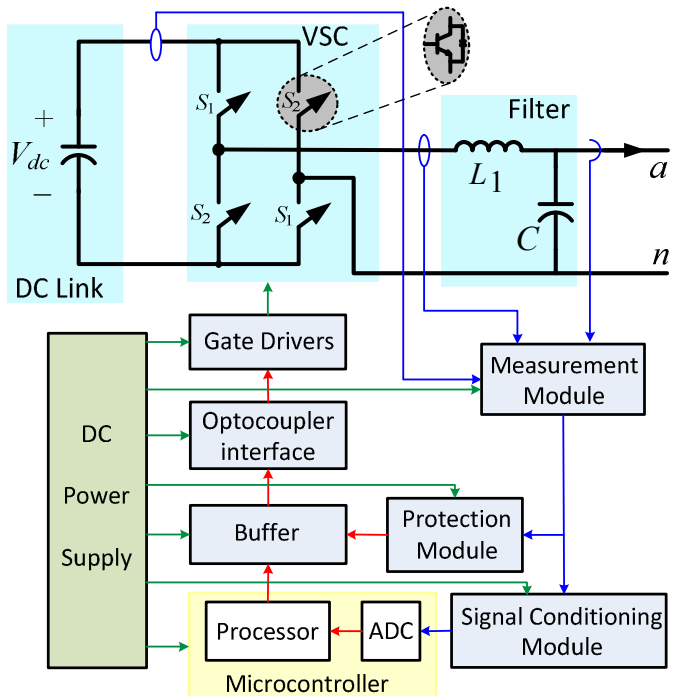

Fig. 1. Schematic diagram of a single-phase H-bridge VSC along with its closed-loop control system.

fabricating the setup and testing. Inevitably, it is risky to work in an electrical laboratory rather than working on a computer with some simulation software packages. In addition to all the previous reasons, the lack of knowledge and unavailability of proper tutorials and guidelines for developing hardware prototypes is another possible reason behind the limited hardware verification.

The majority of the postgraduate students working in power electronics, especially on VSCs, need to verify their designs on the proposed topology, control or application within their research studies by practical prototype implementation.

Hence these students need to be aware of

- objectives of the practical experiment

- the procedure to build up a circuit

- the required auxiliary circuits/modules

- the different available processors in the market, their features and programming methods

- the safety considerations when working in laboratories

- the selection, purchasing or building the required components and elements of the circuit

- the common technical problems they may encounter and the possible solutions for such problems etc. 
Table 1. Comparison among several microcontrollers, available in market, applicable for VSC control.

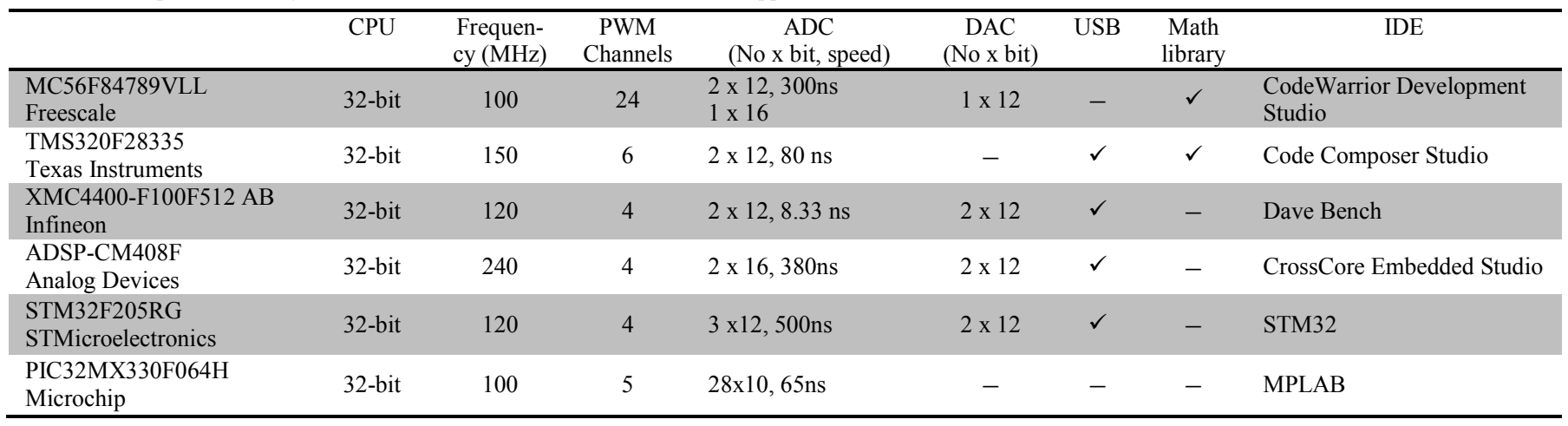

The universities nowadays do not offer units where such skills are taught to the students. Hence, the students can only gather such information from the senior students or build it up based on their personal experience. However, the acquired knowledge is not always transferred properly to the other junior students as the students may not document sufficiently their knowledge by the time they graduate, depriving the university from part of their findings. As a possible outcome, power engineers often limit their goal to demonstrating the experimental success of their proposed design, structure, control or application and may fail to document how they proceeded. Therefore, not much information is provided in the form of tutorials, papers, guidelines, etc. on the steps required to achieve such goals.

Designing and implementing a digitally-controlled VSC requires few basic steps which should be properly planned and organized for a successful completion within the expected time frame. This paper provides a guideline for the beginners who intend to experimentally justify their developed VSC topology, controller or application after simulation studies. The paper also discusses the anticipated administrative and technical difficulties and proposes viable solutions for such difficulties.

The rest of the paper is as follows: Section II describes an example of a VSC system to be implemented practically in the laboratory. This example has been used as a reference through the rest of the paper. Section III discusses the selection and programming of a processor. The guidelines required for the pre-fabrication stage are discussed in Section IV with proper examples. Section V discusses the hardware fabrication stage. The main difficulties of the practical experiments are highlighted in Section VI.

\section{AN EXAMPLE}

To illustrate the guidelines and steps required for implementing a practical prototype VSC better, let us consider a single-phase H-bridge VSC with a topology as shown in Fig. 1. It consists of four Insulated Gate Bipolar Transistors (IGBT), each with an anti-parallel diode. The IGBTs are considered to have proper snubber, driver and protection circuits. The VSC is supplied by a DC link (e.g. a DC power supply or a DC capacitor) while the output of the converter is filtered by a properly tuned LC filter. A voltage-control technique based on bipolar switching of the IGBTs has been adopted for this VSC to provide a desired voltage at the output of its filter. The controller for this VSC is proposed in [5] and is not discussed here as it is beyond the research focus of this paper. In this paper, only the practical aspects and fabrication considerations for practical verification of this controller are discussed in consecutive steps in the following sections.

\section{SELECTING AND PROGRAMMING A PROCESSOR}

Digital controllers are very popular for VSC applications due to their capability to execute complex calculations and flexibility to change the control law with minimum hardware modifications [6]. Implementation of digital controllers is becoming cheap and convenient with the introduction of advanced Micro-Controllers (MC) with enhanced features such as built-in Pulse Width Modulation (PWM) functions and hardware floating point support. Nowadays, there are plenty of MCs available in market with different prices and various features. Hence, selecting a proper $\mathrm{MC}$ for the intended application of VSC control is important. The selected MC should satisfy some requirements such as

- the processor speed,

- the number of Analogue-to-Digital Converters (ADC),

- the number of Digital-to-Analogue Converters (DAC),

- the accuracy,

- the complexity of the calculations and their types

- the amount of memory available

- any special built-in functions/features required such as PWM, External Interface (XINTF), enhanced-Capture (eCAP), Controller Area Network (CAN), Serial Peripheral Interface (SPI), Serial Communications Interface (SCI), Multichannel Buffered Serial Port (McBSP), Inter-Integrated Circuit (I2C), etc.

By comparing the specifications of the different candidate MCs with the system requirements, a suitable MC can be selected considering other features such as the cost, physical dimensions and the technical support available from the manufacturer.

Most of the MC manufacturers provide an Integrated Development Environment (IDE) software, for writing the $\mathrm{C}$ code, compiling the program into assembly language, linking the memory space and downloading the assembly program into the controller. For example, IDE provided by the Microchip for the PIC and dsPIC MCs is named as MPLAB whereas the IDE for the MCs provide by Texas Instrument is 
called Code Composer Studio (CCS). Table 1 also includes the relevant IDEs for different MCs. Alternatively, majority of the MCs can be programmed indirectly through MATLAB/ Simulink using Mathworks Embedded Coder toolbox [7]. A general overview and guideline for programming a MC for VSC control application is provided in [8].

\section{GUIDELINES FOR PRE-FABRICATION STAGE}

A few initial steps can be identified in the process of building a hardware test rig. Following steps are required for any practical prototype implementation.

\section{A. Safety Considerations}

Personal and equipment safety is vital in the hardware building process. Students need to make sure that they follow the safety guidelines adapted by the university. As an example, according to the Australian Standard on Safety in Laboratories Planning and Operational Aspects [9], the maximum Extra-Low Voltage (ELV) in the laboratories is 50 VAC and 120 VDC. Additionally, the universities may have adopted their own laboratory safety guidelines such as restricted access hours, working conditions (such as the requirement of the presence of a supervisor in the laboratory) in addition to the usual safety precautions such as wearing proper clothes, covered safety shoes, safety goggles, gloves, etc.

To practically implement the laboratory prototype of the VSC in Fig. 1, based on the above safety guidelines, the DC link voltage is selected as $100 \mathrm{~V}$ while the $\mathrm{AC}$ voltage at the output of the VSC filter is selected as 40 Vrms. A time schedule is also arranged with the laboratory technician to conduct the laboratory tests. Additionally, the aforementioned general safety precautions are also followed all the time.

\section{B. Selecting the proper Power Electronic Switch}

The main building block of a VSC is the power electronic switch. Bipolar Junction Transistors (BJT), Metal Oxide Semiconductor Field Effect Transistors (MOSFET), Thyristors, Gate Turn-Off Thyristors (GTO) and IGBTs are the most common power electronic switches. Variety of these switches are available in market with different power, voltage and current ratings, switching frequencies, power losses, efficiencies, etc. These switches usually come either as discrete elements (Fig. 2a) or as integrated modules (Fig. 2b). Some of them are even available in the form of Intelligent Power Modules (IPM) with built in driver and protection circuits (Fig. 2c). Discrete components are preferred if a new topology is to be investigated by the student while IPMs are preferred if the main objective of the practical experiment is to verify a new control or application. It is to be noted that IPM modules are relatively expensive compared to the discrete elements however, the discrete elements need separate driver circuits which need to be built by the student.

As the main intention of the experiment is to develop a controller for the VSC in Fig. 1, the PM50CL1A060 IPM, manufactured by Powerex Semiconductor Solutions, is selected (Fig. 2c). This IPM has the maximum tolerable current of $50 \mathrm{~A}$ and voltage of $600 \mathrm{~V}$ DC.

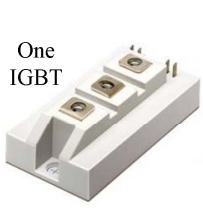

(a)

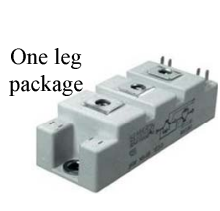

(b)

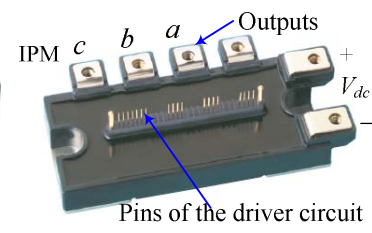

(c)
Fig. 2. IGBT modules as: (a) discrete element, (b) package, (c) IPM.

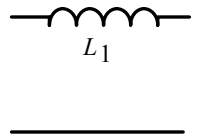

(a)

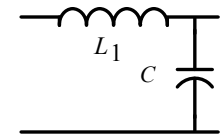

(b)

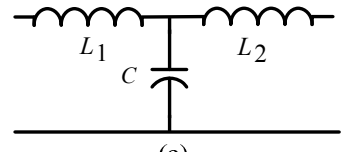

(c)
Fig. 3. Three different passive filters used at VSC outputs.

\section{Designing the VSC Output Filter}

To filter out the switching harmonics from the current and voltage waveforms at the output of the VSC, a passive filter is used. L filters, LC filters and LCL filters are three major filter types used at VSC outputs (Fig. 3). If the VSC is controlled to generate a desired voltage at its output an LC filter is preferred while if controlled to generate a desired current or power at its output an L or LCL filter is preferred. The cutoff frequency of the LC and LCL filter is calculated from

$f_{r}= \begin{cases}\frac{1}{2 \pi} \frac{1}{\sqrt{L_{1} C}} & \text { forLCfilters } \\ \frac{1}{2 \pi} \sqrt{\frac{L_{1}+L_{2}}{L_{1} L_{2} C}} & \text { forLCLfilters }\end{cases}$

where $L_{1}, L_{2}$ and $C$ are the inductance and capacitance of the filter, as shown in Fig. 3.

It is to be noted that the equivalent impedance of the capacitor at fundamental frequency (i.e. $50 \mathrm{~Hz}$ ) should be fairly greater than that of the inductor $L_{1}$. The AC capacitor needs to be purchased from the market. A capacitor with lower Equivalent Series Resistance (ESR) is preferred. If the designed capacitor from (1) is relatively large, the output voltage will be harmonic free; however, the current drawn by the capacitor will be relatively high. This can limit the maximum output current of the VSC and filter system.

The inductors can be purchased or built in the laboratory. To build an inductor, a conductor, preferably one with a higher cross-section (i.e. higher quality factor of $Q=L \omega / R$ ), should be wound with a specific number of turns around a core. A detailed discussion is provided in [10] on the steps of designing an inductor for power electronic applications. This design procedure is beyond the focus of this paper and is not discussed here.

For the VSC system of Fig. 1, a capacitor of $120 \mu \mathrm{F}$ which can tolerate $400 \mathrm{~V} \mathrm{AC} \mathrm{(Fig.} \mathrm{4a)} \mathrm{is} \mathrm{purchased} \mathrm{while} \mathrm{an} \mathrm{induc-}$ tor of $1.5 \mathrm{mH}$ (Fig. 4b) is built on a UU-shape Ferrite core. The inductor and capacitor provide a cut-off frequency of 375 $\mathrm{Hz}$.

D. Selecting and Designing the Auxiliary Modules/Circuits

The schematic diagram of a VSC along with its closedloop control system is shown in Fig. 1. As it is seen from this 
figure, several auxiliary modules are required in the closedloop control system of a VSC. Sensors, filters, level-shifters, amplifiers, processors, buffers, optocouplers and protection circuits are the main auxiliary modules required apart from the power circuit. All these modules need DC power supplies. For building such auxiliary modules, various analog as well as digital integrated circuit (IC) components, voltage and current transducers, discrete electronic components such as resistors, capacitors and operational amplifiers are needed. Hence, their datasheet and application notes need to be referred in order to select the right components. It is to be noted that, more practical information is available in application notes rather than the datasheet. Except the MC which is discussed earlier, rest of these modules is discussed below in detail:

\section{D.1 Sensors}

For the control of a VSC, the currents and voltages of the VSC and its filter need to be monitored and used as the inputs for the VSC controller which is loaded within the MC. Hence, these voltages and currents should be measured through voltage and current transducers.

To implement the VSC measurement module of Fig. 1, the output voltage is measured using the voltage sensor LEM LV 25-P [11], with the equivalent model shown in Fig. 5(a).

Voltage transducers, such as LEM LV 25-P, are relatively very expensive compared to the other auxiliary modules. An alternative method is to use a circuit with a voltage divider and differential amplifier. As an example, the output voltage can be measured using the differential amplifier AD629, with the equivalent model shown in Fig. 5(b). This is a very costeffective method when a large number of voltage measurements are required.

To measure the required currents in the VSC system shown in Fig. 1, a LEM HAIS 50-P current transducer is utilized [12]. The equivalent model of this sensor is shown in Fig. 5(c). This is a hall-effect transducer. One of the main criteria to be considered is the accuracy of its measurements. To measure the current at a specific part of the circuit, the conductor passing that current should be passed through the sensor. To improve the accuracy of the sensor, the conductor may be wound several turns through the sensor. This will increase the precision when a small current is measured. However, it is to be noted that proper number of turns should be calculated as high number of turns may lead to saturation of the sensor. In general, for a sensor with a rating of $x \mathrm{~A}$ to measure a current of $y \mathrm{~A}$ with high precision, the number of turns of the conductor $(n)$ through the current transducer needs to be equal to

$n=\frac{x}{y}$

The output of the current sensor is a voltage that is proportional to the input current. As an example, for the VSC of Fig. 1 , since the expected output current is $5 \mathrm{~A}$, the required number of turns of the conductor wound through the sensor is calculated from (2) as 10 turns.

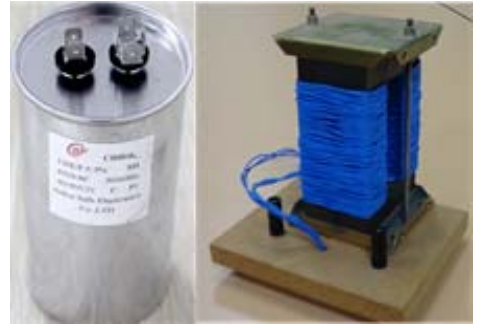

(a)

(b)

Fig. 4. Passive LC filter components at VSC output: (a) $120 \mu \mathrm{F}$ capacitor, (b) $1.5 \mathrm{mH}$ inductor.

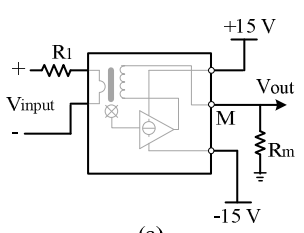

(a)

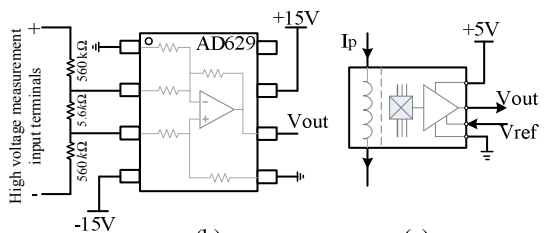

(b)

(c)
Fig. 5. Equivalent model of: (a) LEM LV 25-P voltage transducer, (b)

AD629 differential amplifier, (c) LEM HAIS 50-P current transducer.

The datasheet of LEM HAIS 50-P current transducer defines a gain of $0.625 \mathrm{~V} / \mathrm{A}$ when the transducer is used to measure $50 \mathrm{~A}$. Hence, if the input of the transducer is $50 \mathrm{~A}$, its output will be $31.25 \mathrm{~V}$. Since the expected current at the output of the VSC in Fig. 1 is only 5 A, the transducer does not obey the given gain. Through the measurements in the laboratory, it was revealed that the output of the current transducer will be $5.1 \mathrm{~V}$ for the input current of $5 \mathrm{~A}$. Hence, the transducer demonstrates a gain of 5.1/5 $=1.02$ for the considered application. This gain is valid only to 10 turns of the conductor, passing through the current transducer.

It is to be noted that the voltage transducer and the differential amplifier need to be supplied by $\pm 15 \mathrm{~V}$ DC while the current transducer needs to be supplied by $+5 \mathrm{~V}$ DC.

\section{D.2 Anti-aliasing Filters}

The output analog signals from the voltage and current sensors are digitized before they are fed to the MC. ADCs operate at a constant sampling frequency. According to the Shannon theorem [6], only the signals below the half of the sampling frequency can be digitized reliably. The signals with frequency greater than the half of the sampling frequency fold back into the bandwidth of interest and result in erroneous conversion. Hence, the frequency components greater than half of the sampling frequency needs to be filtered out in order to achieve reliable ADC. These filters are called antialiasing filters which can be realized by inverting or noninverting active filters.

The schematic diagram of the anti-aliasing filter built for the signal conditioning module of the VSC and filter structure of Fig. 1, is shown in Fig. 6 along with the level shifting and amplifying circuit which is discussed in the next section. Since the sampling frequency of the ADCs is $25 \mathrm{kHz}$, the cutoff frequency of the anti-aliasing filter is selected as 12.5 $\mathrm{kHz}$.

\section{3 Level-Shifters and Amplifiers}

The output signal of the sensors should be compatible with the processor specification to which they are applied. Hence, 
these signals should be scaled up/down to match with the requirements of the $\mathrm{MC}$. As an example, some MCs do not accept negative voltages as inputs, hence the output signals of the sensors need to be added with an offset voltage to match with the MC requirement. Then, the result should be scaled up/down to be within the acceptable input voltage range of the MC.

For the VSC system of Fig. 1, a level-shifting circuit is built using two $\mu \mathrm{A} 741$ operational amplifiers. Since, the output signals of the voltage sensors are sinusoidal waveforms with $\pm 1.73 \mathrm{~V}$, hence the resistors of this circuit are selected such that the output signal of the sensors are multiplied by -0.58 and then added with an offset of $1.5 \mathrm{~V} \mathrm{DC}$ to make it within the acceptable range of $0-3 \mathrm{~V}$ of the MC. The schematic diagram of the level shifting circuit is shown in Fig. 6 and its implementation on a veroboard is shown in Fig. 7.

\section{D.4 Blanking Time Function}

To prevent short-circuiting the DC link of the VSC, two switches on the same leg of the VSC should not be conducting at the same time. Hence, the command to turn on a switch should be applied only after the proper turn off of other switch in the same leg. Although the turning off transient period of the switches is few nano to micro seconds, they can vary from one to another. Hence, to guarantee a time difference between turning off of the first switch and turning on of the second switch, a blanking time should be introduced to the turn on gate pulses of the switches. The required blanking time can be introduced to the gate signals through the program within the $\mathrm{MC}$ which is more convenient. The flowchart of the blanking time

For the VSC system of Fig. 1 where the PM50CL1A060 IPM is selected, based on its datasheet, a blanking time of 2 $\mu \mathrm{s}$ is suggested. Hence, a blanking time of $3 \mu \mathrm{s}$ is introduced through the program within the $\mathrm{MC}$.

send Turn off command to one switch; count Time;

if (Time $\geq 3 \mu \mathrm{s}$ ), then (send Turn on command to the other

switch);

Alternatively this blanking time can be introduced by developing an auxiliary circuit between the MC output and the buffers. This approach is not discussed in this paper.

\section{D.5 Buffers}

The MC generates the switching commands in its output pins in digital HIGH and LOW format. These signals should be applied to the driver circuits of the switches through optocouplers. It is to be noted that optocouplers draw some current and hence may load the MC. To prevent this, a buffer circuit should be used between the MC outputs and the optocouplers. Additionally, the buffer circuit can provide logic level shifting function if the input and the output of the buffer are at two different logic levels.

As an example, Fig. 8 shows the equivalent model of the implemented buffer circuit for the control circuit of VSC system of Fig. 1. The buffer is realized using an SN7407 operational amplifier. The output signals from the $\mathrm{MC}$ are at CMOS logic level (i.e. $3.3 \mathrm{~V}$ ) whereas the inputs for the

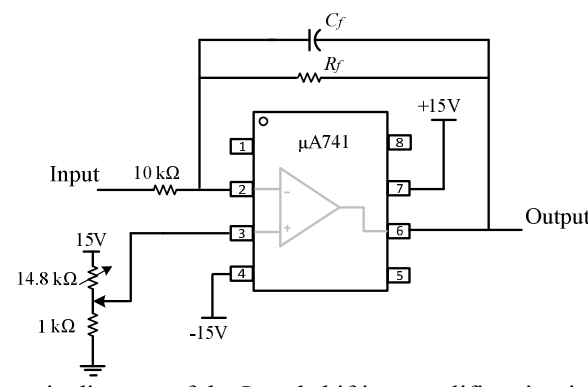

Fig. 6. Schematic diagram of the Level shifting amplifier circuit with single pole inverting anti-aliasing active filter

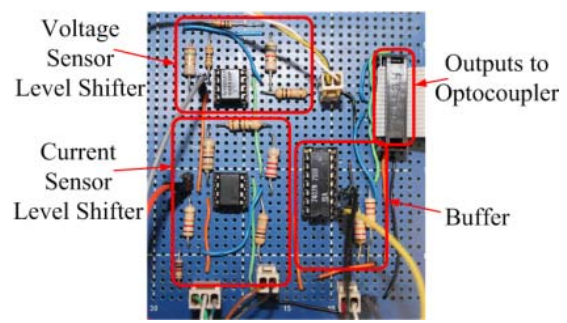

Fig. 7. Implementation of Level-shifting and amplifier circuit con veroborad.

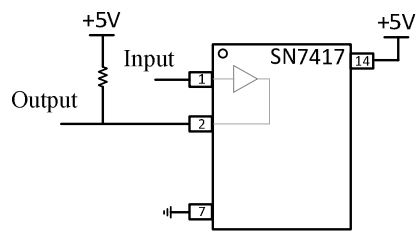

Fig. 8. Equivalent model of buffer.

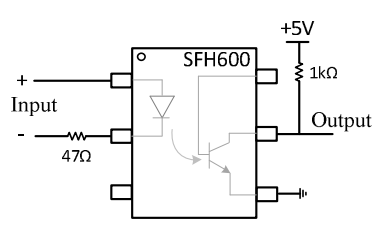

(a)

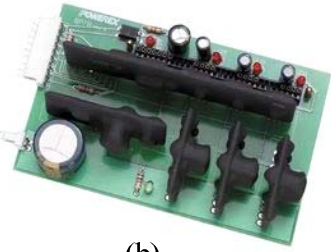

(b)
Fig. 9. (a) Equivalent model of the SFH600 optocoupler, (b) BP7B-LS optocoupler interface.

optocoupler interface are at TTL logic level $(5 \mathrm{~V})$. Hence, in addition to buffering the signals, it provides the logic level shifting operation, too.

\section{D.6 Optocouplers}

Optocouplers are used to electronically isolate the $\mathrm{MC}$ from the power circuit to protect the $\mathrm{MC}$ from probable high voltage damages from the power circuit. Additionally, optocouplers provide transient immunity, too. However, the optocouplers must be fast enough to handle the frequency of the gate signals. Otherwise, it can lead to delays to the turning on and turning off of the switches.

When working with discrete switches, the auxiliary circuits should be built by according to the datasheet of the switches. As an example, SFH600 optocoupler can be utilized in such cases. This optocoupler has a fast response and its equivalent model is shown in Fig. 9(a).

One of the main advantages of utilizing IPMs is that their manufacturers usually provide compatible interface modules such as optocoupler module. For the VSC system of Fig. 1, 
based on the application note of the selected PM50CL1A060 IPM, BP7B-LS optocoupler interface is required (Fig. 9b).

\section{D.7 DC Power Supplies}

All the auxiliary modules need isolated DC power supplies for their operation. These power supplies can be developed by using multi-winding step down transformers and regulator Integrated Circuits (IC). However, it requires time and effort to produce a precisely regulated noise-free DC power supply. Nowadays, small-scale and cheap DC power supplies are available in the market.

For the auxiliary modules of the VSC system of Fig. 1, the $\pm 5, \pm 15$ and +24 V DC are required. Hence, one PS-25-24 power supply and one PT-65C power supply (Fig. 10) are used. They are both supplied by a single-phase $230 \mathrm{~V}$ input and the first one generates \pm 5 and $\pm 15 \mathrm{~V} \mathrm{DC}$ at the output while the second one generates $+24 \mathrm{~V} \mathrm{DC}[13,14]$. It is to be noted that a $10 \mu \mathrm{F}$ capacitor is suggested to be utilized at the inputs of the DC supply for every auxiliary module to eliminate the voltage ripples.

\section{8 Protection Circuit of the DC link}

It is highly important to prevent short-circuiting the DC link, due to probable simultaneous turning on of the two switches in the same leg of the VSC. The protection system can be in the form of an overcurrent protection scheme. Implementing software-based overcurrent protection is not recommended due to its lower reliability.

For the VSC system of Fig. 1, the schematic diagram of the developed overcurrent protection circuit is shown in Fig. 11. The current in the DC link is monitored using a current transducer. The voltage signal coming from this current transducer is compared (using LM339) with a reference to detect the overcurrent condition. The reference voltages of the comparators are set such that they give logic LOW signal in an event of an overcurrent. This reference value is set as 1.25 times of the nominal current. Hence, in the event of an overcurrent, the output of the comparator becomes logic LOW and hence the mono-stable circuit generates a logic HIGH signal at the output. This HIGH signal remains unchanged until it is reset manually by a push button. When the push button is in the OFF position, it generates logic HIGH signal and when it is in $\mathrm{ON}$ position, it generates logic LOW signal. The outputs of the mono-stable circuit and that of the push button are sent through logic NOR gate (MC14001B) in order to derive the protection signal. The MC output signals and the protection circuit output signal are passed through logic AND gate (MC14081B) before being applied to optocoupler interface.

Another alternative method is to energize the DC link with a DC power supply with higher output voltage and current ratings, equipped with current limiting capability to energize the DC link of the experimental prototype.

\section{HARDWARE FABRICATION}

\section{A. Assembling the initial circuit}

After selecting the required hardware elements for the VSC prototype, auxiliary circuits such as signal conditioning circuit and the buffer circuit can be assembled on a breadboard.

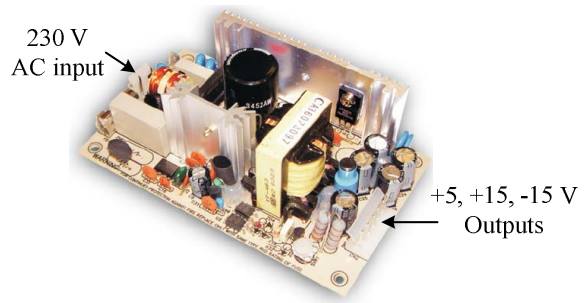

Fig. 10. PT-65C DC power supply.

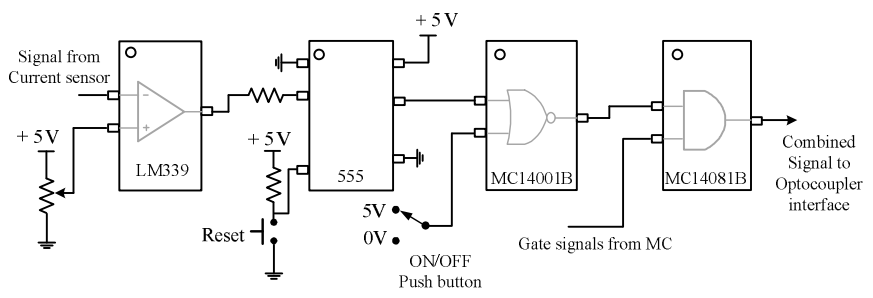

Fig. 11. Schematic diagram of the protection circuit detecting the shortcircuit in the DC link.

It is always suggested that, if possible, the VSC and its filter system is first tested using a DC power supply with inbuilt current limiting feature. This is better than utilizing a battery or an available DC voltage output from photovoltaic, fuel cell, wind turbine etc. After the system is successfully tested, the desired DC supply can be applied instead of the DC power supply, providing that the described DC link protection is already implemented.

It is also suggested that the MC performance to be monitored real-time using its IDE or MATLAB/Simulink.

After successful tests of the system with this initial circuit, the next step is building a veroboard to have all the elements and circuits assembled in order to reduce the effects of stray capacitance due to long wires. After the circuits built on veroboards are successfully tested, a proper Printed Circuit Board (PCB) needs to be designed.

\section{$B$. Designing the $P C B$}

Several software are available in market for PCB design. The full version of popular commercial software like Eagle or Altium might be too expensive and their limited versions may restrict the size and complexity of the board. Free alternatives exist like ExpressPCB, gEDA, DesignSpark, FreePCB, KiCad. For large and complex boards, KiCad is a very good solution as it is free, without restriction on PCB size nor number of layers [15]. The user can draw a schematic of the board and generate the layout automatically. It is recommended to place and route the components manually, even though it can be done automatically with customized settings. The designed layout for the PCB from any PCB design software is called Gerber files which should be sent to PCB manufacturers.

For the auxiliary modules of the VSC system of Fig. 1, the PCB layout is designed using Kicad, as shown in Fig. 12. To make a compact design for the VSC auxiliary circuits, a portion of the $\mathrm{PCB}$ is reserved for mounting the required $\mathrm{MC}$ and the power supplies. 


\section{Printed Circuit Board Assembly (PCBA)}

Manufacturing the PCB requires a budget and time which can cause delays in the practical verification process. It may be expensive to get a PCB manufactured by professional local companies in some countries such as Australia. Alternatively, universities are not always well-equipped for mounting sophisticated components such as surface mounted ICs and components having closer pins. Hence, a possible good solution to save time and money is to contact PCB manufacturers in China where the cost of PCB manufacturing can be as low as a few dollars and as fast as 3-5 days. Aalibaba.com website provides a list of PCB manufacturers which can be communicated to receive quotations for PCB manufacturing [16].

PCBs can be manufactured with either only one conducting layer or with several (2-38) layers. The most common types of the PCBs, which have the cheapest settings and are wellsuited for VSC applications, have the specifications as listed in Table 2.

During the PCB manufacturing process, the holes required for soldering the elements on the PCB need to be plated. Such holes are referred to as Plated through Holes (PTH). The simple mounting holes on the PCB do not need to be plated. Such holes are referred to as non-Plated through Holes (NPTH).

Some manufacturers can also provide the service of mounting the required components on the manufactured PCB. This option is more beneficial for students than buying the components individually from a professional retail shop and mounting them on the PCBs by themselves.

The manufactured PCB with professional PCBA carried out for the VSC system of Fig. 1 is shown in Fig. 13.

\section{DifFICULTIES IN PRACTICAL EXPERIMENTS}

It must be noted that the fabrication of the hardware should be properly planned in order to complete it successfully within the expected time frame. It is also to be noted that university students and researchers have restricted working conditions that can adversely affect the progress of the project. These constraints can be such as laboratory access hours and certain safety rules and regulations that are adapted by the universities. Moreover, there might be some delivery delay if the required components are ordered through the university; hence ordering them well in advance might be necessary.

There are numerous manufacturers and suppliers who provide similar components with different prices. Texas Instruments, Analog devices, Agilent, Semikron and Mitsubishi are some of the well-known manufacturers. Their products can be ordered directly through their websites or through some famous distributors like DigiKey, Element14 and RS Components [1719]. It is advisable to make sure that the right component is being ordered since various models of the same component may be available with different features and ratings. Hence, the datasheet and the relevant application notes need to be carefully checked to avoid the selection of non-suitable components.

Another software-related difficulty in a laboratory prototype experiment is being familiar with the programming languages and the IDE for the selected MC. In this regard, the

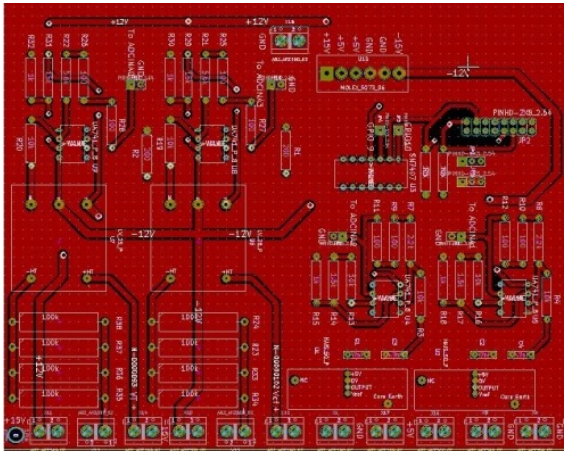

Fig. 12. PCB layout designed for the auxiliary modules of the VSC system of Fig. 1.

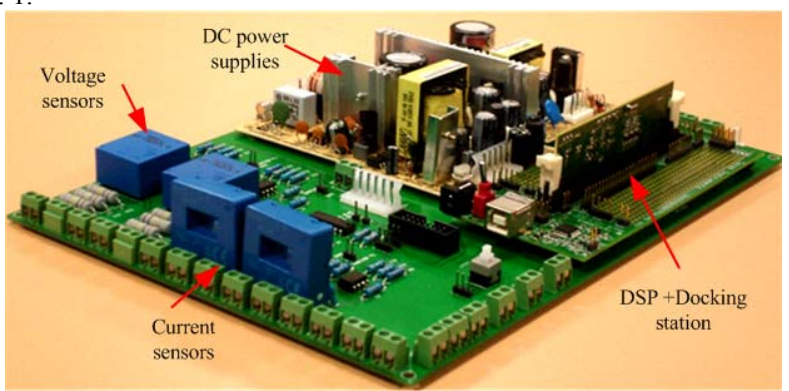

Fig. 13. PCB developed for the VSC system of Fig. 1
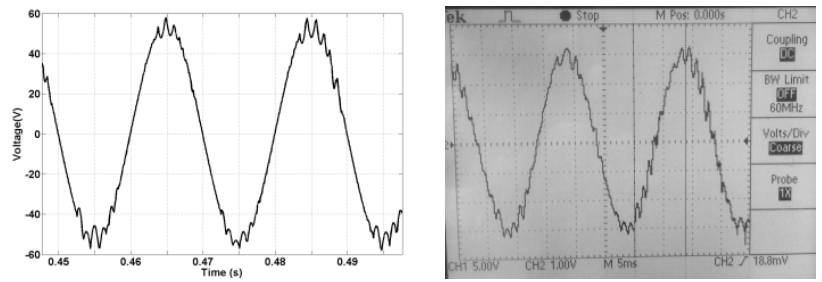

Fig. 14. (a) Simulation results and (b) practical results captured for the VSC system of Fig. 1.

Table 2. Specifications of PCB which are suitable for VSC applications.

\begin{tabular}{ll}
\hline Material & FR4 \\
Board Thickness & $1.6 \mathrm{~mm}$ \\
Copper Thickness & $1 \mathrm{oz}$ \\
Surface treatment & lead free HASL \\
Silkscreen & white \\
Solder mask color & green \\
Legend color & white \\
\hline
\end{tabular}

developer should consider the memory available in each MC, as the memory limitation is a major restriction in developing large and complex programs. Hence, the developed projects should always be customized and optimized to be fit within the available memory of the MC.

After verification by running the software on a preliminary setup, the PCB must be then designed. If KiCad is used, even though this software is free and open-source, the default libraries contain a limited number of components and the user needs to set up the required libraries. This has to be accomplished by using libraries shared by others on Internet, converting schematic symbols, footprints and 3D models from the manufacturers' website, or creating them based on the information from the datasheets.

The simulation and practical test results captured for the VSC system of Fig. 1 are shown in Fig. 14. 


\section{CONCLUSION}

A survey carried out on the literature on voltage source converters revealed that the number of publications with experimental verifications is very limited. Lack of having a scholarly article with proper guidelines for building an experimental hardware prototype is one of the main contributors for above outcome. This paper provides the general guidelines for building a digitally controlled hardware test rig for experimental verification of the VSC control strategies for a beginner. The various steps identified in the process are highlighted with an example hardware building exercise.

\section{REFERENCES}

[1] J. Svensson and R. Ottersten, "Shunt active filtering of vector currentcontrolled VSC at a moderate switching frequency," IEEE Trans. on Industrial Application, Vol. 35, no. 5, pp. 1083-1090, Oct. 1999.

[2] L Wang, H.W. Li and C.T. Wu, "Stability Analysis of an Integrated Offshore Wind and Seashore Wave Farm Fed to a Power Grid Using a Unified Power Flow Controller," IEEE Trans. on Power Systems, Vol. 28, no. 3, pp. 2211-2221, Aug. 2013.

[3] N. Prabhu and K.R. Padiyar, "Investigation of Subsynchronous Resonance With VSC-Based HVDC Transmission Systems," IEEE Trans. on Power Delivery, Vol. 24, no. 1, pp. 433-440, Jan. 2009.

[4] C.A. Sepulveda, J.A. Munoz, J.R. Espinoza, et al. "FPGA v/s DSP Performance Comparison for a VSC-Based STATCOM Control Application," IEEE Trans. on Industrial Information, Vol. 9, no. 3, pp. 13511360, Aug. 2013.

[5] F. Shahnia, R. P. S. Chandrasena, S. Rajakaruna and A. Ghosh, "Primary Control Level of Parallel Distributed Energy Resources Converters in System of Multiple Interconnected Autonomous Microgrids within SelfHealing Networks," IET Generation, Transmission.\& Distribution, Vol. 8, no. 2, pp. 203-222, Feb. 2014.
[6] S. Buso and M. Paolo, Digital Control in Power Electronics. Morgan and Claypool, 2006.

[7] Mathworks Embedded Coder Toolbox, http://www.mathworks.com.au/products/embedded-coder/?s_cid=wiki_simulink_4

[8] F. Barbieri, R.P.S. Chandrasena, F. Shahnia, A. Ghosh and S. Rajakaruna, "Application Notes and Recommendations on Using TMS320 F28335 Digital Signal Processor to Control Voltage Source Converters," Australasian Universities Power Engineering Conference (AUPEC), 2014.

[9] Postgrads OHYG-Laboratory Safety, http://www.uq.edu.au/ohs/pdfs/OHYG-LaboratorySafety.pdf

[10]N. Mohan, T. M. Undeland, and W.P. Robbins, Power Electronics Converters, Applications, and Design, John Wiley \& Sons, 2003.

[11] Voltage Transducer LEM LV 25-P datasheet, Nov. 2012 http://www.lem.com/docs/products/lv\%2025-p.pdf

[12] Current Transducer LEM HAIS 50-P datasheet 100507 http://www.lem.com/docs/products/hais e rev11.pdf

[13] 65W Triple Output Switching Power Supply PS-65 series, doc. ref. PT65-SPEC, Mean Well, 2007. http://www.jameco.com/Jameco/Products/ProdDS/216726.pdf

[14]25W Single Output Switching Power Supply PS-25 series, doc. ref. PS25-SPEC, Mean Well, 2007. http://www.jameco.com/Jameco/Products/ProdDS/392826.pdf

[15]D. Jahshan, P. Hutchinson, F. Tappero, and C. Jarron, "Getting Started in Kicad," 2011.

http://www.kicad-pcb.org/display/KICAD/KiCad+Documentation

[16] http://www.alibaba.com/

[17] http://www.digikey.com/

[18] http://www.element14.com/

[19] http://www.rs-components.com/index.html 